\title{
Optimal Operation of Batch Processes via the Tracking of Active Constraints
}

\author{
Dominique Bonvin and Bala Srinivasan \\ Institut d'Automatique \\ Ecole Polytechnique Fédérale de Lausanne \\ CH-1015 Lausanne, Switzerland \\ dominique.bonvin@epfl.ch
}

November 15, 2001

\begin{abstract}
This paper presents a new measurement-based optimization framework for batch processes, whereby optimal operation is achieved via the tracking of active constraints. It is shown that, under mild assumptions and to a first-order approximation, tracking the necessary conditions of optimality is equivalent to tracking active constraints (both during the batch and at the end of the batch). Thus, the optimal input trajectories can be adjusted using measurements without the use of a model of the process. When only batchend measurements are available, the proposed method leads itself to an efficient batch-to-batch optimization scheme. The approach is illustrated via the simulation of a semi-batch reactor under uncertainty.
\end{abstract}

Keywords: Batch chemical industry, Batch process, Dynamic optimization, Optimal control, On-line optimization, Batch-to-batch optimization, Run-to-run optimization.

\section{Introduction}

Batch and semi-batch processes are of considerable importance in the chemical industry. This paper considers batch and semi-batch processes in the same manner and, thus herein, the term 'batch processes' includes semi-batch processes as well. A wide variety of specialty chemicals, pharmaceutical products, and certain types of polymers are manufactured in batch operations. Batch processes are typically used when the production volumes are low, when isolation is required for reasons of sterility or safety, and when frequent changeovers are necessary. With the recent trend in building small flexible plants that are close to the markets of consumption, there has been a renewed interest in batch processing (Macchietto 1998, Wiederkehr 1988).

Batch chemical processing includes the important steps of capacity planning, tasks scheduling, and operation of individual units (Rippin 1989). This paper considers only the last step which is important for reducing production costs, improving product quality, and meeting safety requirements and environmental regulations. The operation of batch processes typically involves following recipes that have been developed in the laboratory in such a way that they can be implemented safely in production (Friedrich and Perne 1995). However, since there are constraints related to both the operation (equipment limitations, limits on key variables such as temperature or pressure) and the final quality, the operators will naturally introduce a certain conservatism to guarantee feasibility in the presence of process disturbances and differences in equipment and scale. 
To reduce this conservatism, and thereby the operating costs, an optimization approach can be used for recipe adjustment, where the operational decisions such as temperature or feed rate profiles are determined as the solution of an optimization problem. Optimal trajectories are best determined on the basis of a dynamic model of the process (Terwiesch et al. 1994). However, since the models currently available in industry are poor and carry a large amount of uncertainty, standard model-based optimization techniques are by and large ineffective (Bonvin 1998).

This paper presents a scheme for batch process optimization that does not rely on a model for calculating the optimal input trajectories. Instead, it uses a few process measurements in order to track references that guarantee optimal operation, even in the presence of disturbances. The novelty of the approach lies in the fact that the necessary conditions of optimality are used for tracking. Under mild assumptions, the optimization problem can be transformed into a constraint control problem that may include two types of constraints (constraints during the batch and constraints at the end of the batch).

The paper is organized as follows. The industrial practice prevailing in the batch chemical industry and related to optimization is presented in Section 2. Section 3 develops the measurement-based optimization framework from the necessary conditions of optimality and proposes a tracking scheme for its implementation. The approach is illustrated via a simulated example in Section 4. Finally, conclusions are drawn in Section 5 .

\section{Industrial Practice in Batch Processing}

\subsection{Operational Objectives as an Optimization Problem}

The fundamental processing objective is clearly of economic nature, and the optimal operation can be expressed as the solution of an optimization problem with appropriate cost function (Guntern et al. 1998). Performance improvement for processing systems often pushes the process towards constraints that are related to equipment and operational limitations and to safety aspects. In batch processing, there is the additional effect of constraints at the end of the batch (selectivity in reaction systems, purity in separation systems, admissible levels of impurities, etc.). A typical optimization problem includes some of the following elements:

- A cost function to optimize

1. Productivity: Since productivity can be expressed in many ways, it is chosen here as the net profit of a given production campaign. Reducing the time necessary for a given production is particularly interesting when the number of batches per shift can be increased.

2. Time-to-market: The economic performance is strongly tied to the speed at which a new product/process can be developed. Since production in campaigns reduces the time to learn, it is necessary to learn quickly and improve the productivity right away.

- Constraints during the batch

1. Safety aspects: The safety aspects such as the avoidance of runaways are very important. Safety requirements can lead to highly conservative operation, especially for slow and exothermic reactions.

2. Batch variability: High productivity calls for stable production so as to reduce the amount of corrective manual operations that are costly in terms of production time and personnel. This requires reduction of batch variability caused, for example, by on-line disturbances and batch-tobatch raw material variations. This aspect is important when the process has to work closely to some quality limit. 
1. Product quality: Quality is often impaired by the appearance of small amounts of undesired byproducts. The presence of impurities is very critical since it can turn an acceptable product into waste. Removing impurities at the end of the batch is often not possible or prohibitively expensive and can significantly reduce throughput. In such cases, the selectivity objectives become critical.

2. Environmental impact: The amount and toxicity of by-products, together with the amount and environmental costs of raw materials, are important factors as well.

\subsection{Optimization in Industry}

Though the formulation of the operational objectives as an optimization problem is fairly straightforward, there have been only a few attempts in industry to optimize operations through mathematical modeling and optimization techniques. The reasons are briefly discussed next.

Standard optimization methods rely heavily on a dynamic model of the process. It is illusory to expect constructing detailed kinetic models since the molecules treated in the batch industry are typically more complex than in the commodity industry and often result in complicated reaction pathways. The development of dynamic models may exceed one man-year, which is incompatible with the objectives of batch processing. Also, the processes are subject to variations that cannot be predicted in advance.

The common industrial practice to cope with this uncertainty is to design an operating policy that can meet the desired specifications in most cases. Thus, such a strategy is quite conservative in nature. In an optimization problem with constraints (safety, operational limitations, etc.), a conservative strategy implies process operation far from the constraints in order to reduce the danger of violating them. Due to these large safety margins, the performance can be quite poor.

However, with the advent of more measurements, a framework that relies on measurements rather than on an accurate model of the process for reducing the conservatism introduced in process operation can be used. Nowadays, quality measurements are typically available at the end of the batch via, for example, off-line chromatographic methods. In addition, physical measurements such as temperature, flow, pressure, or $\mathrm{pH}$ are routinely available on-line during the course of the batch. Other on-line measurements such as conductivity, viscosity, refractive index, torque, spectroscopy, and calorimetry are readily available in the laboratory and are now used increasingly in production as well.

An interesting feature of batch processing is the fact that batch processes are repeated over time. Thus, the operation of the current batch can also be improved by using the measurements available from previous batches. In this case, the objective is to reach optimal operation over as few batches as possible in a so-called batch-to-batch optimization scheme (Srinivasan et al. 2001a).

\section{Measurement-based Optimization}

Process control is a field where measurements are used either to keep the process at desired set points despite perturbations or to follow desired reference trajectories. Importance is given to designing a controller that is capable of: i) bringing the process to the desired trajectory and maintaining it therein (stability), ii) rejecting the disturbances that occur in the system (disturbance rejection), and iii) guaranteeing performance despite variations in the system dynamics (robustness and/or adaptation). In all these studies, the reference trajectories are considered to be given.

However, designing reference trajectories is a task by itself and should take into account process performance. 
One way of addressing the performance objectives is through optimization. Thus, the main question is: "How can the references be chosen so that the desired cost be optimized?". Or, in other words: "How can an optimization problem be transformed into a control problem?".

The standard approach consists of choosing a set of variables (mostly, variables that can be measured easily on-line) and compute their optimal time evolutions using a model of the process. These evolutions are then provided as the reference trajectories to track using feedback control. The main difficulty with this approach is the inevitable presence of uncertainty in any industrial setting (in the form of disturbances and modeling errors). Due to uncertainty, the optimal evolutions of the references change and thus adaptation of the reference trajectories is required.

An adaptation approach that has been used extensively in the literature is the indirect (or model-based) adaptation scheme (Rawlings et al. 1989, Ruppen et al. 1998). This consists of refining the model (i.e., re-identifying the model parameters with fresh data) and using the refined model to recompute the reference trajectories. The major drawbacks of this approach are: i) computational complexity due to the fact that both the parameter estimation and the optimization have to be repeated, and ii) the excitation provided by the optimal inputs may not be rich enough to uncover the model parameters.

The approach used in this paper is a direct one that does not require a model of the process for adaptation. In the previous approach, the variables to track were chosen based on how easily they can be measured. In contrast here, they are chosen such that their reference trajectories are not affected by disturbances. The choice of these variables, which is based on the necessary conditions of optimality, represents the novelty in the proposed methodology.

\subsection{Necessary Conditions of Optimality}

This subsection presents the mathematical formulation and justification for transforming an optimization problem into that of tracking active constraints. In the next subsections, a scheme for tracking active constraints is developed.

Consider the class of optimization problems that is appropriate for dealing with industrially-relevant batch processes, i.e., dynamic optimization with a final-time cost function and both path and terminal constraints. The dynamic optimization problem can be formulated mathematically as follows (Kirk 1970, Bryson and Ho 1975):

$$
\begin{aligned}
\min _{u(t)} J= & \phi\left(x\left(t_{f}\right)\right) \\
\text { s.t. } & \dot{x}=F(x, u, \theta), \quad x(0)=x_{0} \\
& S(x, u, \theta) \leq 0, \quad T\left(x\left(t_{f}\right), \theta\right) \leq 0
\end{aligned}
$$

where $J$ is the scalar performance index to be minimized at the (free or fixed) final time $t_{f}, x$ the states with known initial conditions $x_{0}, u$ the inputs, $S$ the path constraints, $T$ the terminal constraints, $F$ the system equations, $\theta$ the parameters of the system, and $\phi$ the terminal cost.

Note that the dynamic optimization problem (1)-(3) has two types of constraints: i) the path constraints $S(x(t), u(t), \theta)$ that impose bounds on the inputs and the states during the batch, and ii) the terminal constraints $T\left(x\left(t_{f}\right), \theta\right)$ that limit the outcome of the batch at final time. The necessary conditions of optimality are given by (Bryson and Ho 1975): 


\begin{tabular}{|l|l|l|}
\hline & Path & Terminal \\
\hline Constraints & $\mu^{T} S(x, u, \theta)=0$ & $\nu^{T} T\left(x\left(t_{f}\right), \theta\right)=0$ \\
\hline Sensitivities & $\lambda^{T} \frac{\partial F}{\partial u}+\mu^{T} \frac{\partial S}{\partial u}=0$ & $\lambda^{T}\left(t_{f}\right)-\left.\frac{\partial \phi}{\partial x}\right|_{t_{f}}-\left.\nu^{T}\left(\frac{\partial T}{\partial x}\right)\right|_{t_{f}}=0$ \\
\hline
\end{tabular}

where $\lambda(t)$ are the adjoint variables (Lagrange multipliers for the system equations), $\mu(t)$ the Lagrange multipliers for the path constraints, and $\nu$ the Lagrange multipliers for the terminal constraints.

Three important conclusions can be drawn:

- Since the necessary conditions of optimality (4) remain valid for any value of $\theta$, they represent expressions whose trajectories are not affected by uncertainty. Moreover, the reference values for these expressions are trivially equal to zero (see Equation 4).

- The necessary conditions of optimality have two parts: i) the constraint part (first row of 4), and (ii) the sensitivity part (second row of 4).

- Both the constraint and sensitivity parts have two elements: i) the path elements corresponding to quantities during the run (first column of 4), and ii) the terminal elements related to quantities at the end of the run (second column of 4).

\subsection{Tracking the Necessary Conditions of Optimality}

These conclusions suggest tracking the necessary conditions of optimality. However, the Lagrange multipliers $\lambda, \mu$, and $\nu$ are neither measurable nor straightforward to compute. To make the problem easier to deal with, the inputs can be parameterized and certain (reasonable) assumptions introduced.

\section{- Piecewise parameterization of the inputs}

The constraint part of the necessary conditions of optimality implies that some of the path and terminal constraints will be active in the optimal solution. Let denote these active path and terminal constraints by $S_{a}$ and $T_{a}$, respectively. The way the input parameterization is performed depends on the number of inputs.

1. In the single-input case, the input trajectory is divided into several time intervals, each interval being characterized by an active path constraint, $\left(S_{a}\right)_{i}=0$. In the example presented in Section 4 , the optimal input profile $u(t)$ depicted in Figure 2 exhibits three intervals characterized by the upper bound $u_{\max }$, the safety part $u_{\text {safety }}$ and the lower bound $u_{\min }$, respectively. In an interval with an active path constraint, the input is adjusted to keep the path constraint active (this is the case with all three intervals in Figure 2). In an interval with no active path constraint, the input can be approximated by a polynomial. The coefficients of all the polynomial approximations, along with the lengths of the various intervals, are referred to as the 'input parameters' and will be adapted to meet the active terminal constraints, $T_{a}=0$.

2. The multi-input case is a bit more complicated. With several inputs, there can be more inputs than active path constraints in a given interval. Then, some combinations of the inputs will be used to keep the path constraints active, while the other inputs are approximated using polynomials (Srinivasan et al. 2002).

\section{- Assumptions for tracking}

1. Assumption 1: Assume that the active (path and terminal) constraints do not change with uncertainty. This means that, though the lengths of the intervals can vary, the active path constraints in each interval and the sequence of intervals should be invariant under uncertainty. Furthermore, this assumption implies that the active terminal constraints remain the same. 
2. Assumption 2: For an input to be optimal, the necessary conditions (4) need to be satisfied. Any uncertainty will cause a deviation from the active constraints or from zeroing the sensitivities. It can be argued that a deviation from an active constraint is typically more costly than a deviation from a zero sensitivity. Indeed, in a Taylor-series expansion of the cost, the cost variation due to the constraints not being active represents a first-order term, while the sensitivities have a second-order effect. Thus, if it can be assumed that the uncertainties are small enough for the first-order effects to dominate the higher-order ones, there is much more to be gained by keeping the path and terminal constraints active compared to tracking the sensitivities to zero.

Under these two assumptions, the problem of tracking the necessary conditions of optimality reduces to that of tracking the active constraints. Notice that, though the necessary conditions depend on a model of the process, no model is necessary once the problem has been formulated as a constraint tracking problem. To ensure optimality, the deviations from the active constraints simply need to be reduced to zero using measurements.

\subsection{Description of the Tracking Scheme}

Though tracking constraints for the sake of performance has been studied since the early 1970s (Maarleveld and Rijnsdorp 1970), the present formulation exhibits certain peculiarities. First of all, the optimal solution consists of several intervals, with the active constraints being different for each interval. Furthermore, the input parameters described in Section 3.2 are used to handle terminal constraints.

The proposed tracking scheme, which is labeled 'Measurement-based Optimization' (MBO), involves the following two parts (Figure 1):

- Path controllers: A path controller (such a terminology is used since it tracks an active path constraint) is designed for each active path constraint in a given interval. For implementation, on-line measurements of the deviations from the constraints are required, i.e., measurements during the run. The controllers are typically PI-type controllers that can be designed using any of the standard techniques.

- Terminal controllers: The terminal constraints are satisfied by adjusting the input parameters. Adjustment is performed on a run-to-run basis, exploiting the fact that batch runs are typically repeated. The deviations from the terminal constraints are measured at the end of the run and corrected using PI-type terminal controllers (such a terminology is used since they track the terminal constraints).

In Figure 1, the trajectory generator synthesizes the inputs $u(t)$ from: i) the input parameter values obtained from the terminal controllers, and ii) bits of inputs obtained from the path controllers. With this scheme, if the structure proposed for the optimal solution (type and sequence of intervals, active terminal constraints) is correct, simple feedback controllers will push the system towards the optimal input trajectories $u_{o p t}(t)$.

\subsection{Practical Applicability of MBO}

Certain issues regarding the applicability of $\mathrm{MBO}$ in practice are briefly discussed next.

- Role of a model: The active path and terminal constraints and the structure of the optimal solution (i.e., the various intervals that constitute the solution) can be determined via numerical optimization based on a simplified (or tendency) model. The purpose of a model is only to provide the correct structure of the optimal inputs, and not to determine the optimal inputs themselves. Hence, in contrast to model-based optimization approaches, there is no need for a detailed model or for accurate parameter values. No model is required if the educated guess of an experienced operator is sufficient to provide the structure of the optimal solution. 


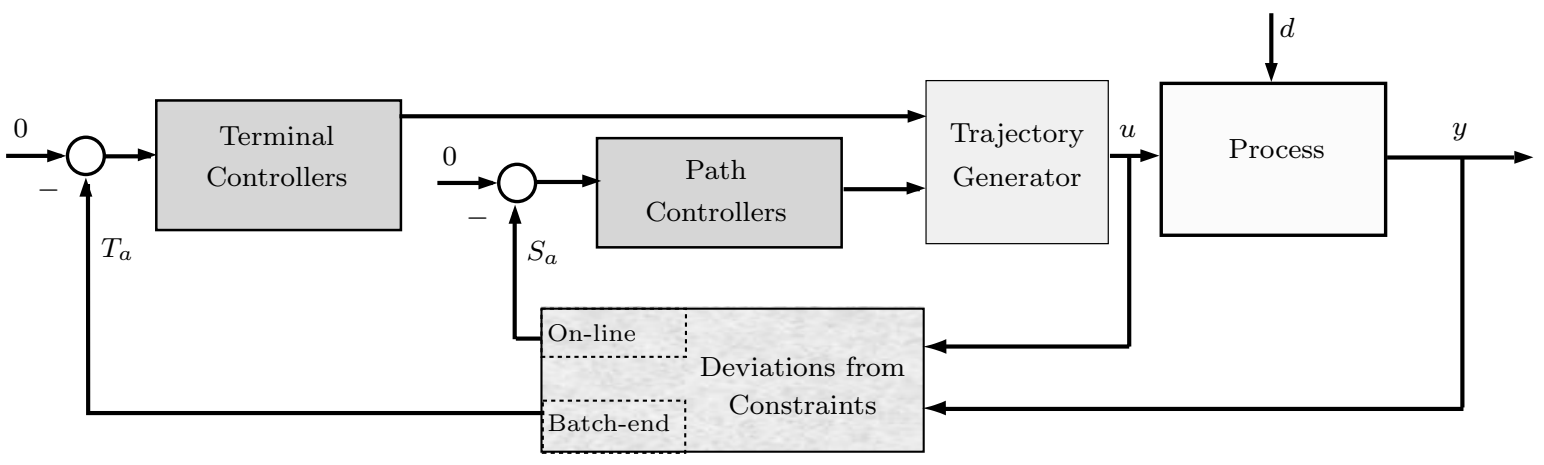

Figure 1: Measurement-based optimization via tracking of the active constraints $S_{a}=0$ and $T_{a}=0$

- Deviations from constraints: Often, the deviations from the active path and terminal constraints can be obtained directly from on-line and batch-end measurements, respectively. Also, in some cases, a path constraint can be rewritten in terms of quantities that can be easily measured. For example, a constraint on the rate of heat removal in an exothermic reactor can be rewritten as a constraint on the cooling temperature as will be illustrated in the example of next section. When this is not possible, some type of inference or state estimation is necessary, which clearly complicates the approach.

- Difference in time scale - on-line vs. batch-end measurements: In general, there is a difference in time scale between the path controllers and the terminal controllers. The path controllers work within a batch using on-line measurements (the running index is the batch time $t$ ). The terminal controllers operate on a batch-to-batch basis using batch-end measurements (the running index is the batch number $k$ ). If on-line measurements are not available, the path controllers are inactive, thereby leading to a simple batch-to-batch optimization scheme.

- Disturbance rejection and backoffs from constraints: Since control action is never perfect in dynamic systems, the regulated active constraints $S_{a}$ and $T_{a}$ are not zero at all time. Furthermore, if only batch-end measurements are used in a run-to-run scheme, the effect of any disturbance within the batch cannot be rejected. In such cases, the use of conservative margins for the set points, called backoffs, is necessary to ensure that the resulting batch performance is indeed feasible, i.e., it does not violate the operational and quality constraints (Visser et al. 2000). The presence of measurement errors also calls for backoffs.

- Optimal solution on the constraints: Constraints play an important role in the optimization problem and, in many cases, the optimal solution is determined by the path and terminal constraints. Thus, once the type and sequence of intervals are chosen and the lengths of the intervals adjusted to meet the path and terminal constraints, the solution is optimal. In such a case, Assumption 2 mentioned in Section 3.2 is trivially satisfied since there are no sensitivities that need to be driven to zero. The optimal solution being on the constraints is a fairly common property of the problems considered in the batch chemical industry.

\section{Example - Semi-batch Reactor with Safety and Purity Con- straints}

\subsection{Description of the Reaction System}

- Reaction: $A+B \rightarrow C$. 
- Conditions: Semi-batch through feeding of B, isothermal.

- Objective: Maximize the quantity of $C$ produced at a given final time.

- Input variable: Feed rate of $B$.

- Constraints: Upper and lower bounds on the input, upper limit on the rate of heat removal through the jacket (safety constraint), upper limit on the amount of $B$ at final time (purity constraint).

- Comments: The reactor is kept isothermal at temperature $T$ by (say) adjusting the cooling temperature in the jacket, $T_{c}$. $B$ is fed at the feed temperature $T_{i n}$ and it is assumed that $T_{i n}=T$. To remain isothermal, the power generated by the reaction, $q_{r x}$, must be evacuated through the cooling jacket, i.e., $q_{r x}=U A\left(T-T_{c}\right)$. Thus, the upper limit on the heat removal rate can be expressed as a lower limit on the cooling temperature, $T_{c m i n}$. The variables and parameters are described in the next subsection.

Without the constraints, optimal operation would simply consist of adding all the available $B$ at the initial time (i.e., batch operation). However, the heat removal constraint limits the initial amount of $B$ in the reactor and thus calls for semi-batch operation. Furthermore, the feed rate of $B$ has to be reduced to its minimum after a certain time to meet the terminal constraint regarding the amount of $B$ left at final time.

\subsection{Problem Formulation}

Variables and parameters: $c_{X}$ : Concentration of species $\mathrm{X}, n_{X}$ : Number of moles of species $\mathrm{X}, V$ : Reactor volume, $u$ : Feed rate of $\mathrm{B}, c_{B i n}$ : Inlet concentration of $\mathrm{B}, k$ : Kinetic parameter, $\Delta H$ : Reaction enthalpy, $T$ : Reactor temperature, $T_{c}$ : Cooling temperature in the jacket, $T_{i n}$ : Feed temperature, $U$ : Heat transfer coefficient, $A$ : Surface for heat transfer, and $q_{r x}$ : power produced by the reaction.

Model equations:

$$
\begin{array}{rlrl}
\dot{c_{A}} & =-k c_{A} c_{B}-\frac{u}{V} c_{A} & c_{A}(0) & =c_{A_{o}} \\
\dot{c_{B}} & =-k c_{A} c_{B}+\frac{u}{V}\left(c_{B i n}-c_{B}\right) & c_{B}(0) & =c_{B o} \\
\dot{V} & =u & V(0) & =V_{o}
\end{array}
$$

The amount of $C$ is given by

$$
n_{C}=c_{c} V=c_{A o} V_{o}-c_{A} V
$$

The power produced by the reaction is given by $q_{r x}=(-\Delta H) k c_{A} c_{B} V$, and the cooling temperature necessary to keep the reactor isothermal can be expressed as

$$
T_{c}=T-\frac{q_{r x}}{U A}=T-(-\Delta H) \frac{k c_{A} c_{B} V}{U A} .
$$

The numerical values are listed in Table 1.

\begin{tabular}{|lcc|}
\hline$k$ & 0.75 & $\frac{1}{\mathrm{molh}}$ \\
$\Delta H$ & $-7 \times 10^{4}$ & $\frac{\mathrm{J}}{\mathrm{mol}}$ \\
$U A$ & $8 \times 10^{5}$ & $\mathrm{~J} / \mathrm{Kh}$ \\
\hline
\end{tabular}

\begin{tabular}{|lcc|}
\hline$T$ & 35 & ${ }^{\circ} \mathrm{C}$ \\
$t_{f}$ & 2.5 & $\mathrm{~h}$ \\
$c_{B \text { in }}$ & 10 & $\frac{\mathrm{mol}}{\mathrm{l}}$ \\
\hline
\end{tabular}

\begin{tabular}{|lcc|}
\hline$u_{\min }$ & 0 & $\frac{1}{\mathrm{~h}}$ \\
$u_{\max }$ & 100 & $\frac{1}{\mathrm{~h}}$ \\
$T_{c_{\min }}$ & 10 & ${ }^{\circ} \mathrm{C}$ \\
$n_{B f_{\max }}$ & 100 & $\mathrm{~mol}$ \\
\hline
\end{tabular}

\begin{tabular}{|lcc|}
\hline$c_{A o}$ & 2 & $\mathrm{~mol}$ \\
$c_{B o}$ & 0 & $\mathrm{~mol}$ \\
$V_{o}$ & 500 & $\mathrm{l}$ \\
\hline
\end{tabular}

Table 1: Model parameters, operating conditions, bounds, and initial conditions 
Optimization problem:

$$
\begin{array}{cll}
\max _{u(t)} & J=n_{C}\left(t_{f}\right) \\
\text { s.t. } & (5)-(9) & \\
& T_{c}(t) \geq T_{c \min } \quad \text { (safety) } \\
& n_{B}\left(t_{f}\right) \leq n_{B f_{\max }} \quad \text { (purity) } \\
& u_{\min } \leq u(t) \leq u_{\max }
\end{array}
$$

\subsection{Determination of the Structure of the Optimal Solution}

Sequence of intervals: Numerical solution of Problem (10) shows that the optimal input trajectory consists of three intervals (Figure 2):

- $B$ is first fed at the rate $u_{\max }$ until $T_{c}(t)=T_{c m i n}$, which determines the first switching time $t_{s_{1}}$.

- Then, $u_{\text {safety }}(t)$ is applied to keep $T_{c}(t)=T_{c m i n}$.

- At time instant $t_{s_{2}}$, the input is set to $u_{\min }$ so as to be able to meet the terminal constraint $n_{B}\left(t_{f}\right)=$ $n_{B f_{\max }}$.

The corresponding evolutions of the amounts of $A, B$, and $C$ and of the reactor and cooling temperatures are given in Figures $3-4$. For the numerical values provided in Table $1, t_{s_{1}}=0.25 \mathrm{~h}, t_{s_{2}}=1.18 \mathrm{~h}$ and $J=486.8 \mathrm{~mol}$.

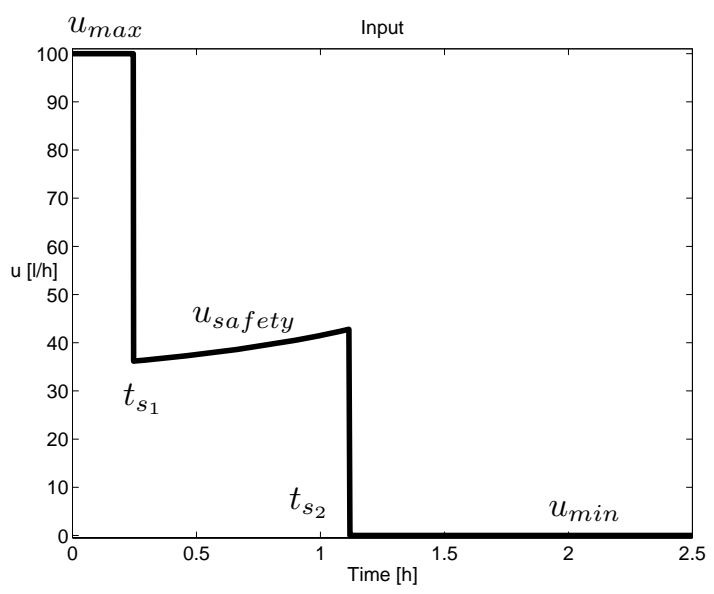

Figure 2: Optimal input

Effect of different problem formulations:

1. If the safety constraint were not there, it would be optimal to continue feeding at the maximum rate, and reduce the feed to $u_{\min }$ only to meet the purity constraint at final time. Such a strategy would lead to $J=525.6 \mathrm{~mol}$. So, a loss in productivity of $7 \%$ is the "price" that has to be paid for safety.

2. Without the terminal constraint on $n_{B}\left(t_{f}\right)$, the optimal solution would correspond to feeding $B$ according to $u_{\text {safety }}(t)$ until the final time $t_{f}$. Since more $B$ could be added this way, the quantity of $C$ produced would be $J=683.5 \mathrm{~mol}$ ( $40 \%$ more).

Dealing with uncertainty: In practice, there can be considerable uncertainty in the stoichiometric and kinetic models (possibility of side reactions, unknown rate constants, etc.). This is reflected here as some uncertainty 


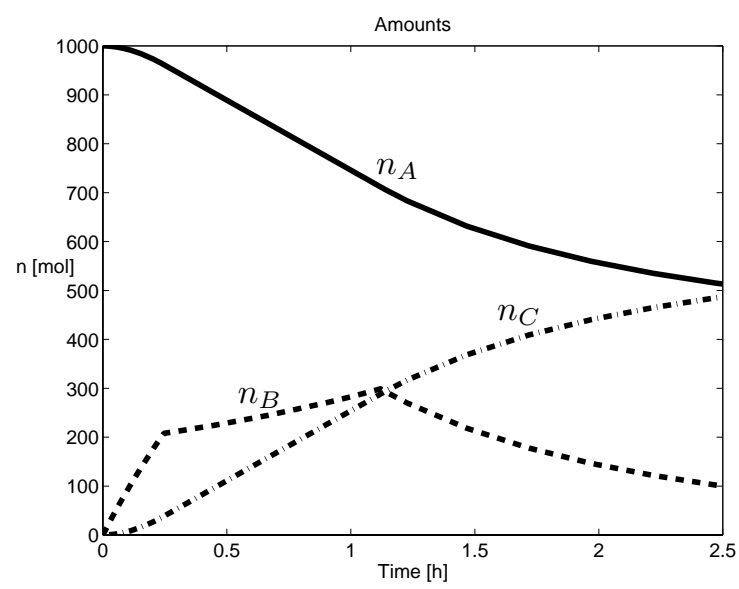

Figure 3: Evolution of the numbers of moles

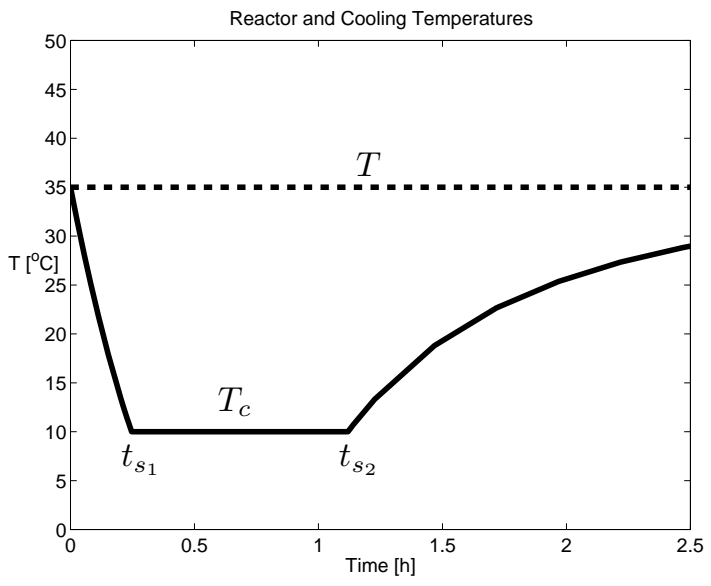

Figure 4: Evolution of the reactor and cooling temperatures

in the kinetic parameter $k$ in the range $0.4 \leq k \leq 1.2$ (the nominal value $k=0.75 \frac{l}{\mathrm{molh}}$ used in the simulation is assumed to be unknown).

It has been verified that, for the uncertainty considered, the qualitative solution remains the same, i.e., the type and sequence of the three intervals are the same as in Figure 2. However, the switching times $t_{s_{1}}$ and $t_{s_{2}}$ and $u_{\text {safety }}(t)$ have to be adjusted to compensate for the uncertainty.

\subsection{Reducing Deviations from Constraints using Measurements}

The switching time $t_{s_{1}}$ and $u_{\text {safety }}(t)$ are adjusted during the batch using the on-line measurement of $T_{c}(t)$ to meet the safety constraint $T_{c_{m i n}}$. The switching time $t_{s_{2}}$ is adjusted on a batch-to-batch basis using the batch-end measurement of $n_{B}\left(t_{f}\right)$ in order to meet the purity constraint $n_{B f_{\max }}$. Measurements with $5 \%$ gaussian noise are considered. Because of the measurement noise, conservative margins (or backoffs) are added to the set points so as to guarantee feasibility ( $-5 \mathrm{~mol}$ for $n_{B f_{\max }}$ and $0.5{ }^{\circ} \mathrm{C}$ for $T_{c_{\min }}$ are chosen here and correspond to the $5 \%$ deviations contributed by measurement noise). Four optimization scenarios are given in Table 2 and are discussed briefly next.

1. No measurement: In order not to violate the constraints, a conservative feed profile is designed to handle the worst-case scenarios: i) the safety constraint is met for $k=k_{\max }=1.2 \frac{l}{\mathrm{molh}}$, and ii) the purity constraint is met for $k=k_{\text {min }}=0.4 \frac{l}{\mathrm{molh}}$. Thus, the conservative profile is obtained from the 


\begin{tabular}{|c|c|c|c|c|c|}
\hline & Optimization Scenario & $\begin{array}{c}\text { Terminal Constraint } \\
n_{B}\left(t_{f}\right) \text { mol } \\
\left(n_{B f_{\max }}=100 \mathrm{~mol}\right)\end{array}$ & $\begin{array}{c}\text { Path Constraint } \\
\min _{t} T_{c}(t)^{\circ} \mathrm{C} \\
\left(T_{c \min }=10^{\circ} C\right) \\
\end{array}$ & $\begin{array}{c}\text { Cost } \\
n_{C}\left(t_{f}\right) \\
(\mathrm{mol}) \\
\end{array}$ & Loss \\
\hline 1 & $\begin{array}{l}\text { No measurement } \\
\text { Open-loop application of the } \\
\text { optimal conservative input }\end{array}$ & 38.7 & 13.5 & 338 & $44 \%$ \\
\hline 2 & $\begin{array}{l}\text { Measurement of } T_{c}(t) \\
\text { Adaptation of } t_{s_{1}} \text { and } u_{\text {safety }}(t) \\
\text { Improvement in a single batch }\end{array}$ & 53.5 & 10.5 & 399 & $22 \%$ \\
\hline 3 & $\begin{array}{l}\text { Measurement of } n_{B}\left(t_{f}\right) \\
\text { Adaptation of } t_{s_{2}} \\
\text { Improvement over } 50 \text { batches }\end{array}$ & 95.0 & 12.5 & 450 & $8.1 \%$ \\
\hline 4 & $\begin{array}{l}\text { Measurements of } T_{c}(t) \text { and } n_{B}\left(t_{f}\right) \\
\text { Adaptation of } t_{s_{1}}, u_{\text {safety }}(t) \text { and } t_{s_{2}} \\
\text { Improvement over } 50 \text { batches }\end{array}$ & 95.0 & 10.5 & 477 & $2.8 \%$ \\
\hline
\end{tabular}

Table 2: Measurement-based optimization. In Cases 2-4, 5\% gaussian noise is added to the measurements and the results are averaged over 100 noise realizations. The backoffs used for the constraint set points are $-5 \mathrm{~mol}$ for $n_{B f_{\max }}$ and $0.5^{\circ} C$ for $T_{c \min }$. The constraints that are met (within the size of the backoff) are indicated in italics.

dynamic model by computing $t_{s_{1}}^{\text {cons }}$ and $u_{\text {safety }}^{\text {cons }}(t)$ so that the path constraint be met for $k=k_{\text {max }}$, and $t_{s_{2}}^{\text {cons }}$ so that the terminal constraint be satisfied for $k=k_{\text {min }}$. As a result, $u_{\text {safety }}^{\text {cons }}(t)$ is a bit lower than that in Figure $2, t_{s_{1}}^{\text {cons }}=0.15 \mathrm{~h}$, and $t_{s_{2}}^{\text {cons }}=0.82 \mathrm{~h}$. This conservative feed profile is applied open loop, without measurements, to the simulated nominal plant. Such a conservative strategy exhibits a $44 \%$ loss in productivity compared to the optimal solution that assumes perfect knowledge of the reaction system.

2. On-line measurement of $T_{c}(t)$ : The safety constraint is kept active (within the size of the backoff) using the feedback law $u_{\text {safety }}(t)=u_{\text {safety }}^{\text {cons }}(t)+k_{p}\left(T_{c m i n}-T_{c}(t)\right)+k_{i} \int_{0}^{t}\left(T_{c m i n}-T_{c}(t)\right) d t$, where $k_{p}$ and $k_{i}$ are the parameters of a PI controller (the path controller of Figure 1). This controller is switched on when $T_{c}(t)=T_{c m i n}$. Thus, $t_{s_{1}}$ is determined implicitly upon reaching $T_{c m i n}$. The second switching time is kept at the conservative value $t_{s_{2}}^{\text {cons }}$ since no batch-to-batch improvement is considered. This simple strategy reduces the loss in productivity to $22 \%$.

3. Batch-end measurement of $n_{B}\left(t_{f}\right)$ : The switching time $t_{s_{2}}$ is updated on a batch-to-batch basis using the terminal controller of Figure 1. The first switching time is kept at its conservative value $t_{s_{1}}^{\text {cons }}$ and, for the second interval, $u_{\text {safety }}^{\text {cons }}(t)$ is applied in an open-loop manner. With this strategy, the loss in productivity reduces significantly to about $8 \%$. The evolution of the cost function for batch-to-batch optimization over 50 batches is shown in Figure 5. Notice that the terminal constraint, and thus also the maximal cost, is reached within a few batches.

4. On-line measurement of $T_{c}(t)$ and batch-end measurement of $n_{B}\left(t_{f}\right)$ : Here, $t_{s_{1}}$ and $u_{\text {safety }}(t)$ are updated within the batch, while $t_{s_{2}}$ is updated on a batch-to-batch basis. This way, both the path and the terminal constraints can be met (within the size of the backoff). Despite measurement errors and unknown kinetic parameter, the loss in productivity is less than $3 \%$ compared to the true optimum. This loss, which results from the backoffs, can be reduced if the measurements are more accurate. This scenario with both on-line and batch-end measurements clearly shows how effective the MBO scheme can be when the optimal operation is indeed on active constraints. 


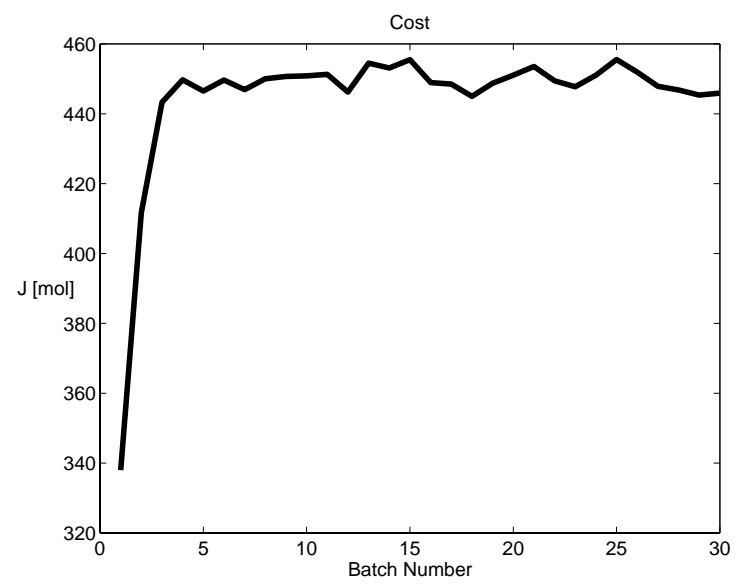

Figure 5: Evolution of the cost (number of moles of $C$ ) for one realization of the batch-to-batch optimization with only batch-end measurement of $n_{B}\left(t_{f}\right)$ ( $5 \%$ measurement noise)

\subsection{Discussion}

The MBO scheme requires no model as long as the assumption that the optimal solution involves the three intervals - first $u_{\max }$, then $u_{\text {safety }}$, and finally $u_{\min }$ - holds. As far as the implementation is concerned, $u_{\max }=100 \mathrm{l} / \mathrm{h}$ and $u_{\min }=0 \mathrm{l} / \mathrm{h}$ are applied in an open-loop manner, while $u_{\text {safety }}(t)$ is determined by a PI-controller upon tracking $T_{c_{m i n}}$. The switching time $t_{s_{1}}$ is determined upon reaching $T_{c m i n}$, while $t_{s_{2}}$ is adjusted on a run-to-run basis by a PI-controller in order to meet $n_{B f_{\max }}$.

Assume that, in addition to the modeled reaction $A+B \rightarrow C$, the true reaction system includes a second reaction from among the following set: $A+B \rightarrow D, C \rightarrow D, A+C \rightarrow D$ and $B+C \rightarrow D$. This would not affect the type and sequence of intervals $\left(u_{\max }\right.$ followed by $u_{\text {safety }}$ and $\left.u_{\min }\right)$ since the additional reaction does not introduce the possibility of independent competition for the reactant $B$ as was shown in (Srinivasan et al. 2001b). Thus, the proposed scheme would apply equally when in the presence of an additional unmodeled reaction.

In the formulation of the optimization problem, $T_{i n}=T$ was assumed. Even without this assumption, the proposed approach is directly applicable. The possibility of removing heat through temperature increase of the feed from $T_{i n}$ to $T$ (so-called sensible heat) modifies the heat removal constraint to:

$$
q_{r x}-q_{i n} \leq U A\left(T-T_{c m i n}\right)
$$

with $q_{\text {in }}$ the rate of heat removal associated with the feeding of $B$. However, the implementation of the heat removal constraint remains unchanged as it concerns only the RHS of $(11): u_{\text {safety }}(t)$ is determined as the output of a PI-controller designed to track $T_{c m i n}$.

As a final remark, it is important to stress that the model parameters given in Table 1 are not used for calculating the optimal feed rate of $B$. Only the batch-end measurement of $n_{B}\left(t_{f}\right)$ is necessary for implementing the batch-to-batch optimization (Scenario 3). The complete MBO scheme (Scenario 4) requires in addition the on-line measurement of $T_{c}(t)$.

\section{Conclusions}

The lack of reliable models and the presence of uncertainty have favored the investigation of process improvement via the utilization of measurements (both on-line and batch-end). The major contribution of 
this paper is to have laid the way for transforming a model-based optimization problem into a model-free control problem. On the basis of a few (reasonable) assumptions and of an appropriate parameterization of the input profiles, optimal operation can be achieved, to a first-order approximation, via the tracking of the active path and terminal constraints. If only off-line measurements are available, this framework results in a batch-to batch optimization scheme capable of meeting the terminal constraints within a few batches. If on-line measurements are available, the path constraints can also be kept active.

The proposed measurement-based optimization scheme addresses most of the industrial requirements mentioned in Section 2.2. More specifically,

- it is model independent as far as the adjustment of the inputs is concerned,

- it is robust against uncertainty since signals that are invariant under uncertainty (the necessary conditions of optimality) are tracked, and

- it is based on both on-line and batch-end measurements in order to meet path and terminal constraints.

It is possible to perceive the proposed measurement-based optimization strategy from an industrial perspective. Classical PID control is the most popular technique used currently in industry, and trading it to attain optimality is unacceptable industrially. Therefore, in contrast to most model-based optimization studies, this work attempts to use measurements and feedback control for the sake of optimality. In this sense, the approach has great industrial potential and should help take optimization to the batch chemical industry.

\section{References}

Bonvin, D. (1998). Optimal operation of batch reactors - A personal view. J. Process Contr. 8(5-6), 355-368.

Bryson, A. E. and Y. C. Ho (1975). Applied Optimal Control. Hemisphere, Washington DC.

Friedrich, M. and R. Perne (1995). Design and control of batch reactors: An industrial viewpoint. Comp. Chem. Eng. 19, S357-S368.

Guntern, C., A. H. Keller and K. Hungerbühler (1998). Economic optimization of an industrial semi-batch reactor applying dynamic programming. Ind. Eng. Chem. Res. 37(10), 4017-22.

Kirk, D. E. (1970). Optimal Control Theory : An Introduction. Prentice-Hall, London.

Maarleveld, A. and J. E. Rijnsdorp (1970). Constraint control on distillation columns. Automatica 6, 51-58.

Macchietto, S. (1998). Batch process engineering revisited: Adding new spice to old recipes. In: IFAC $D Y C O P S-5$. Corfu, Greece.

Rawlings, J. B., N. F. Jerome, J. W. Hamer and T. M. Bruemmer (1989). End-point control in semi-batch chemical reactors. In: IFAC DYCORD+'89. Maastricht, Netherlands. pp. 339-344.

Rippin, D. W. T. (1989). Control of batch processes. In: IFAC DYCORD+'89. Maastricht, Netherlands. pp. $115-125$.

Ruppen, D., D. Bonvin and D. W. T. Rippin (1998). Implementation of adaptive optimal operation for a semi-batch reaction system. Comp. Chem. Eng. 22, 185-189.

Srinivasan, B., C. J Primus, D. Bonvin and N. L. Ricker (2001a). Run-to-run optimization via generalized constraint control. Control Eng. Practice 9, 911-919.

Srinivasan, B., O. Ubrich, D. Bonvin and F. Stoessel (2001b). Optimal feedrate policy for systems with two reactions. In: IFAC DYCOPS-6. Cheju Island, Korea. pp. 455-460. 
Srinivasan, B., S. Palanki and D. Bonvin (2002). Dynamic optimization of batch processes: I. Characterization of the nominal solution. Comp. Chem. Eng. p. Submitted.

Terwiesch, P., M. Agarwal and D. W. T. Rippin (1994). Batch unit optimization with imperfect modeling A survey. J. Process Contr. 4, 238-258.

Visser, E., B. Srinivasan, S. Palanki and D. Bonvin (2000). A feedback-based implementation scheme for batch process optimization. J. Process Contr. 10, 399-410.

Wiederkehr, H. (1988). Examples of process improvements in the fine chemicals industry. Comp. Chem. Eng. 43, 1783-1791. 